\title{
OPTICAL PROPERTIES OF THE RADIO SOURCE B2 0828+32
}

\author{
M.-H. ULRICH-DEMOULIN AND J. RÖNNBACK \\ European Southern Observatory, Karl-Schwarzschild-Strasse 2, \\ D-85748 Garching bei Munchen, FRG
}

A small number of radio galaxies have two sets of classical double radio lobes with the radio axes aligned in different directions. Furthermore differences in the properties of the radio lobes such as the surface brightness and spectral index indicate that the two sets of double radio structure have different ages. The radio ejection axis has therefore changed direction with time. In the first two known radio galaxies of this type, 3C 315 and B2 $0055+26[1]$, the host galaxy is a member of a close pair of ellipticals in a common optical envelope suggesting that the complex radio structure is caused by gravitational interaction $[1,2]$.

We recently took deep CCD images in $\mathrm{R}$ and V of $\mathrm{B2} 0828+32$ which is another example of radio galaxies with two double radio structures. The $\mathrm{R}$ image is shown in Fig. 1. The radio spectral index maps of this radio galaxy allow to set an age to the old pair of radio lobes, 70 Myears, which is thus an upper limit to the age of the young lobes [3]. This is interesting as it constrains the time scale of the phenomenon which changes the direction of the radio axis.

The major results from the study of the optical data can be summarized as follows:

A) The host galaxy of B2 $0828+32$ has no double core or double nucleus, in contrast with $3 \mathrm{C} 315$ and $\mathrm{B} 20055+26$, and it has no close companion brighter than $M_{v}=-16.7$ within $38 \mathrm{kpc}$ (see Fig. 1) assuming $\mathrm{H}_{0}=100$ $\mathrm{km} \mathrm{s}^{-1} \mathrm{Mpc}^{-1}$. It lies at the edge of a poor cluster.

B) The shape of the luminosity profile is typical for early type galaxies with a de Vaucouleurs $\mathbf{r}^{1 / 4}$ profile, but there is some discrepancy between data and model at radii less than $\sim 15$ arcsec. This could be a sign of an old merger (transient shell structures or superposition of two mixed components). The relaxation time for a merger of 2 massive galaxies with rotating bulges is of the order $1 \mathrm{Gyr}$. 


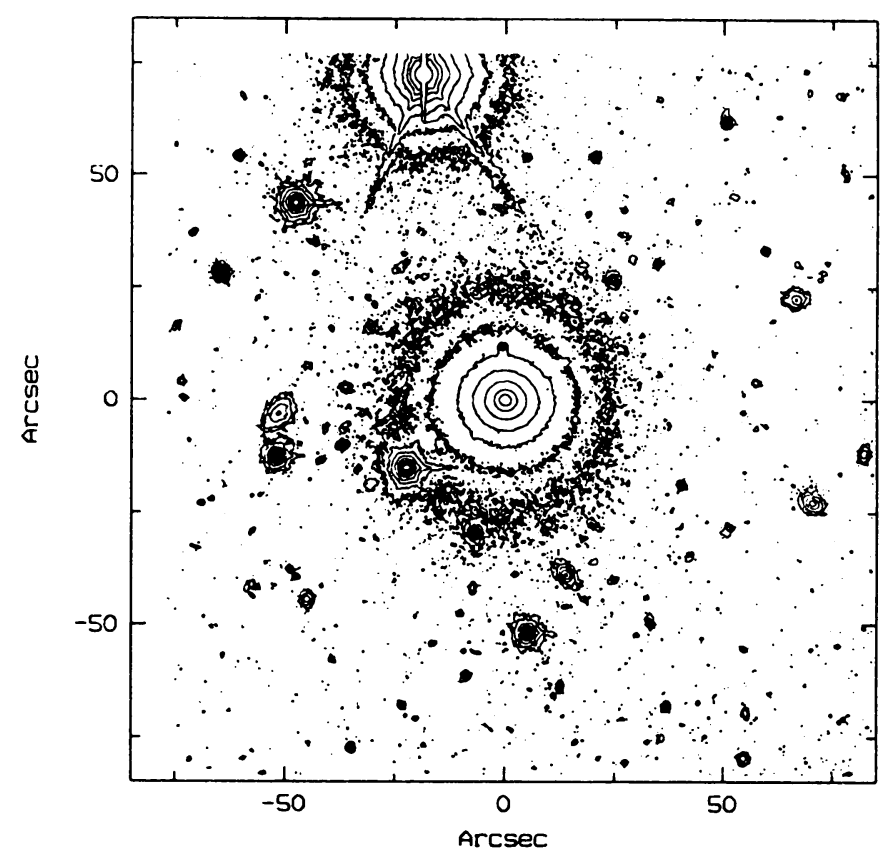

Figure 1. Contour plot in $\mathrm{R}$ of $\mathrm{B} 20828+32$. North to the top and east left.

The integrated $\mathrm{V}-\mathrm{R}$ of the host galaxy is blue, $\mathrm{V}-\mathrm{R}=0.54 \pm 0.06$, being approximately constant as a function of radius between $5^{\prime \prime}$ and $30^{\prime \prime}$. A typical colour for an early type galaxy is $\mathrm{V}-\mathrm{R} \sim 0.6-0.7$.

We conclude that the optical images of B2 $0828+32$ do not show strong signatures of a recent major merger event. However, the blue colours and weak features in the luminosity profile indicating that the stellar distribution has been disturbed dynamically, may be the last remaining signs of a major merger event some 1 Gyr ago.

This leads us to suggest a model where the change of radio axis direction was caused by the merging of a small galaxy [4] whose gas gathered at the center of the host and was accreted by the black hole (generally believed to power the radio source). We have shown that this could occur in less than 70 Myears if the accretion proceeds at a rate close to the Eddington rate [4]. That event ignited and fueled the younger radio lobes.

\section{References}

[1] Ekers, R.D., Fanti, R., Lari, C., Parma, P. 1978, Nature, 276, 588

[2] Parma, P., Ekers, R.D., Fanti, R. 1985, A\&AS, 59, 511

[3] Klein, U., Mack, K.-H., Gregorini, L., Parma, P. 1995, $A \& A$, in press

[4] Ulrich, M.-H., Rönnback, J. 1995, in preparation 